\title{
Desenvolvimento de cookies adicionados de farinha de yacon (Smallanthus sonchifolius): caracterização química e aceitabilidade sensorial entre portadores de Diabetes Mellitus
}

\author{
Yacon (Smallanthussonchifolius) flour added-cookies: chemical characterization \\ and sensory acceptability among the patients with diabetes mellitus
}

RIALA6/1608

\author{
Mayla Gabriely Giacomel RODRIGUES ${ }^{1}$, Elisvânia Freitas dos SANTOS², Fabiane La Flor Ziegler SANCHES², Daiana \\ NOVELLO $^{1 *}$, Maria Raquel MANHANI ${ }^{3}$, Mikael NEUMANN ${ }^{4}$ \\ *Endereço para correspondência: ${ }^{1}$ Departamento de Nutrição, Setor de Ciências da Saúde, Universidade Estadual do Centro- \\ Oeste (UNICENTRO). Caixa Postal 35, CEP: 85010-000, Guarapuava, PR, (42) 3629 8182. E-mail: nutridai@gmail.com. \\ ${ }^{2}$ Departamento de Nutrição, Universidade Federal de Mato Grosso do Sul (UFMS), Campo Grande, MS \\ ${ }^{3}$ Departamento de Farmácia e Nutrição, Universidade São Judas Tadeu, São Paulo, SP \\ ${ }^{4}$ Departamento de Medicina Veterinária, Setor de Ciências Agrárias e Ambientais, Universidade Estadual do Centro-Oeste \\ (UNICENTRO), Guarapuava, PR \\ Recebido: 23.08.2013 - Aceito para publicação: 16.06.2014
}

\section{RESUMO}

O objetivo do trabalho foi verificar a aceitabilidade sensorial de biscoitos tipo cookies adicionados de farinha de yacon (FY) e determinar a composição química da formulação tradicional e daquela contendo o maior teor de FY com aceitação sensorial semelhante ao padrão. Utilizaram-se cinco formulações de biscoitos, sendo: padrão (0 \%) e as demais com $5 \%, 7,5 \%, 10 \%$ e $12,5 \%$ de FY. Na análise sensorial participaram 55 provadores não treinados, portadores de diabetes mellitus de ambos os gêneros com idade entre 18 a 70 anos. Os resultados da análise sensorial mostraram que a amostra com adição de 7,5\% de FY foi o melhor teor de yacon acrescentado e com aceitação semelhante ao padrão, principalmente nos atributos aparência, aroma, sabor, aceitação global e intenção de compra. A análise química das amostras padrão e com 7,5 \% de FY revelou maiores teores de fibra bruta, proteínas, cinzas e umidade na amostra com FY, entretanto apresentou menores concentrações de carboidratos e valor calórico total. A elaboração desses produtos permitiu comprovar que, um nível de adição de até 7,5 \% de FY em biscoitos tipo cookies foi bem aceito pelos provadores, obtendo-se aceitação sensorial semelhante ao produto padrão.

Palavras-chave. índice glicêmico, alimentos funcionais, raízes tuberosas.

\section{ABSTRACT}

This study analyzed the sensory acceptability of yacon flour (YF) added-cookies, and determined the chemical composition of the traditional formulation and of those containing the highest contents of YF with the sensory acceptance similar to the standard product. Five formulations of biscuits have been prepared, being: standard ( $0 \%$ ) and four samples containing $5 \%, 7.5 \%, 10 \%$ and $12.5 \%$ of YF, respectively. For conducting the sensory analysis, 55 untrained tasters participated, who were diabetic patients of both genders, aged from 18 to 70 years. The results of sensory analysis showed that the addition of $7.5 \%$ of YF was the best contents of yacon, and with the acceptance similar to the standard product, and mainly for the attributes appearance, aroma, taste, overall acceptance and purchase intention. The chemical analysis of standard samples and of $7.5 \%$ YF-containing cookies showed higher levels of fiber, protein, ash and moisture in the sample with YF; however lower concentrations of carbohydrates and total calories were found. The development of products has demonstrated that the cookies containing YF up to $7.5 \%$ were well accepted by the tasters and these samples evidenced the sensory acceptance similar to the standard product. Keywords. glycemic index, functional foods, tuberous roots. 
Rodrigues MGG, Santos EF, Sanches FLFZ, Novello D, Manhani MR, Neumann M. Desenvolvimento de cookies adicionados de farinha de yacon (Smallanthus sonchifolius): caracterização química e aceitabilidade sensorial entre portadores de Diabetes Mellitus Rev Inst Adolfo Lutz. São Paulo, 2014; 73(2):219-25.

\section{INTRODUÇÃO}

O Diabetes Mellitus (DM) é um estado de hiperglicemia crônica por deficiência ou produção insuficiente de insulina pelo pâncreas, resultando na incapacidade deste hormônio em exercer suas funções no metabolismo da glicose. Dessa maneira, a terapia nutricional é vital para pacientes diabéticos e tem como principal objetivo manter os níveis de glicemia o mais próximo do normal, juntamente com o uso de medicamentos e a prática e exercícios físicos ${ }^{1}$.

Portadores dessa patologia necessitam de alimentos ricos em fibras, com baixos teores de gordura saturada, sal e açúcares simples. A fibra solúvel favorece o controle de dislipidemias e da glicemia, por reduzir a absorção de colesterol e carboidratos no âmbito intestinal ${ }^{1}$. Sabendo-se disso, o desenvolvimento de novos produtos adicionados de ingredientes funcionais, mostra-se cada vez mais desafiador para o setor alimentício, que visa atender às exigências do mercado consumidor atual, caracterizado pela procura de produtos saudáveis e atrativos. Neste sentido, a batata yacon (Smallanthus sonchifolius) ou "batata diet", pode ser considerada uma opção saudável para o consumo de pacientes portadores de DM. Seu benefício nutricional pode ser obtido pela ingestão in natura ou como ingrediente em produtos normalmente consumidos, uma vez que é classificada como um alimento funcional ${ }^{2}$.

Diferentemente das demais raízes tuberosas que armazenam carboidratos na forma de amido, a yacon possui como principais substâncias de reserva os frutooligossacarídeos (FOS), frutose, glicose e sacarose em menor proporção ${ }^{2}$. Dentre os FOS presentes nessa raiz, encontra-se a inulina, um polímero de frutose, que atravessa o trato digestivo sem ser metabolizada. A inulina apresenta as propriedades das fibras alimentares solúveis, que promovem o crescimento e atividade de bactérias probióticas ${ }^{3}$. Estas fibras estão relacionadas ao aumento da viscosidade do bolo alimentar e, como conseqüência, ao retardo na digestão e absorção de carboidratos, que acarretam a diminuição da hiperglicemia pós-prandial precoce e do risco de hipoglicemia reacional e prolonga o período de saciedade ${ }^{4}$, características benéficas aos pacientes com DM.

Um alimento propício para a adição de novos ingredientes funcionais em sua formulação são os cookies, que apresentam fácil preparo, longa vida de prateleira e boa aceitação por parte da população, sendo, portanto, uma ótima possibilidade de produto para os portadores de DM. Diante do exposto, o objetivo desse trabalho foi produzir cookies contendo farinha de yacon como ingrediente em substituição ao açúcar utilizado na formulação tradicional, os quais foram analisados quanto sua aceitação sensorial e composição química.

\section{MATERIAL E MÉTODOS}

\section{Preparo da farinha de yacon}

Foram adquiridos $10 \mathrm{~kg}$ de yacon (Smallanthus sonchifolius) em um supermercado local da cidade de Guarapuava, PR. As raízes tuberosas foram higienizadas em água corrente e descascadas. A polpa foi submersa em água a $70{ }^{\circ} \mathrm{C}$ por 10 minutos e, então submersa em água a temperatura ambiente por 5 minutos. Em seguida, as polpas $(8,5 \mathrm{~kg})$ foram cortadas em fatias finas, acomodadas em telas (treliça/32 mesh) e dispostas em estufa ventilada com circulação de ar (Pardal', Brasil) para secagem direta por 48 horas a $60{ }^{\circ} \mathrm{C}^{5}$. Após este período, foram trituradas em liquidificador (Philips/Walita, Brasil), obtendo rendimento de $600 \mathrm{~g}$ de farinha, a qual foi acondicionada em potes hermeticamente fechados.

\section{Formulações dos cookies}

Foram elaboradas cinco formulações de cookies, sendo: F1: padrão ( $0 \%$ de FY) e as demais adicionadas de 5,0 \% (F2), 7,5 \% (F3), 10,0 \% (F4) e 12,5\% (F5) de FY. Estes níveis de adição foram definidos por meio de testes sensoriais preliminares realizados com o produto.

Além das porcentagens citadas de FY, os ingredientes utilizados nas formulações foram: farinha de trigo refinado (52,9\%), açúcar cristal (F1: 26,4 \%, F2: 21,4 \%, F3: 18,9 \%, F4: 16,4 \%, F5: 13,9 \%), ovos (13,2 \%), margarina vegetal (5,59\%), fermento em pó $(1,32 \%)$ e sal refinado $(0,44 \%)$.

Os produtos foram elaborados, individualmente, no Laboratório de Técnica Dietética do Departamento de Nutrição da UNICENTRO, Guarapuava, PR. Primeiramente, foram separados os ingredientes: farinha de trigo, açúcar cristal, ovos, margarina, sal, fermento em pó e FY (conforme cada nível de adição). Os ingredientes foram misturados pela ordem citada, até obter uma massa grossa e homogênea. Em seguida, a massa foi moldada em formato de cookies (com aproximadamente $40 \mathrm{~mm}$ de diâmetro), dispostos em formas de alumínio e assados em forno (Arno , Brasil) pré-aquecido por $200^{\circ} \mathrm{C}$ durante 20 minutos. Cada cookie apresentou peso aproximado de $30 \mathrm{~g}$. 
Rodrigues MGG, Santos EF, Sanches FLFZ, Novello D, Manhani MR, Neumann M. Desenvolvimento de cookies adicionados de farinha de yacon (Smallanthus sonchifolius): caracterização química e aceitabilidade sensorial entre portadores de Diabetes Mellitus Rev Inst Adolfo Lutz. São Paulo, 2014; 73(2):219-25.

\section{Análise sensorial}

Participaram da pesquisa 55 pacientes portadores de Diabetes Mellitus, cadastrados no Programa HiperDia do Sistema Único de Saúde do Município de Guarapuava, PR. Os provadores foram de ambos os gêneros, não treinados, com idade entre 18 a 70 anos.

A avaliação sensorial foi realizada em uma sala própria, localizada junto ao local dos encontros mensais, em cabines individuais, sob luz natural.

Foram avaliados os atributos de aparência, aroma, sabor, textura e cor, por meio de uma escala hedônica estruturada de 9 pontos, variando de desgostei muitíssimo (nota 1) a gostei muitíssimo (nota 9). Foram aplicadas também, questões de aceitação global com auxílio de escala hedônica estruturada de 9 pontos (1: desgostei muitíssimo a 9: gostei muitíssimo) e de intenção de compra, por escala estruturada de 5 pontos 1: certamente não compraria a 5: certamente compraria) $)^{6}$.

Um teste de ordenação foi aplicado para comparar as diferenças das amostras para o atributo específico de sabor doce, já que os cookies foram reduzidos em açúcar. Nesse teste, os provadores classificaram as cinco amostras em ordem crescente de doçura, sendo da menos doce para a mais doce ${ }^{6}$.

Os julgadores receberam uma porção de cada amostra (aproximadamente $30 \mathrm{~g}$, um biscoito), em pratos plásticos brancos codificados com números de três dígitos, de forma balanceada e casualizada, acompanhada de água para realização do branco. As formulações foram oferecidas de forma monádica sequencial.

\section{Índice de aceitabilidade (IA)}

O cálculo do índice de aceitabilidade das cinco formulações foi realizado conforme Dutcoski ${ }^{6}$, segundo a fórmula: $I A(\%)=A \times 100 / B$, onde $A=$ nota média obtida para o produto; $B=$ nota máxima dada ao produto.

\section{Análises químicas}

As análises químicas foram realizadas no Laboratório de Nutrição Animal do Departamento de Medicina Veterinária da UNICENTRO Guarapuava, PR. As seguintes determinações foram realizadas, em triplicata, na formulação padrão e naquela com maior nível de adição de FY e com aceitação sensorial semelhante a padrão:

Determinação da umidade: Foi realizada de acordo com Instituto Adolfo Lutz, que consiste em secagem a $105^{\circ} \mathrm{C}$ até peso constante.

Determinação de cinzas: A porcentagem de cinzas foi determinada em mufla a $550{ }^{\circ} \mathrm{C}^{5}$. Determinação de proteínas: Foi realizada através da avaliação do nitrogênio total da amostra, pelo método Kjeldahl determinado ao nível semimicro ${ }^{5}$. Utilizou-se o fator de conversão de nitrogênio para proteína de 6,25.

Determinação de lipídios: As amostras foram avaliadas pelo método de extração a frio ${ }^{7}$. Determinação de carboidratos: A determinação de carboidratos dos produtos foi realizada por meio de cálculo teórico (por diferença), conforme a fórmula: \% Carboidratos $=100-$ (\% umidade $+\%$ proteína $+\%$ lipídios $+\%$ cinzas $+\%$ fibra alimentar).

Determinação de fibra bruta: A fibra bruta foi avaliada conforme metodologia do Instituto Adolfo Lutz .

Determinação do valor calórico total: $\mathrm{O}$ total de calorias (kcal) foi calculado utilizando os seguintes valores: para lipídios $(8,37 \mathrm{kcal} / \mathrm{g})$, proteína $(3,87 \mathrm{kcal} / \mathrm{g})$ e carboidratos $(4,11 \mathrm{kcal} / \mathrm{g})^{8}$.

\section{Determinação do Valor Diário de Referência (VD)}

O VD foi calculado em relação a $30 \mathrm{~g}$ da amostra ${ }^{9}$, com base nos valores médios preconizados para adultos e idosos, conforme gênero e idade para adultos (18 a 70 anos) e para idosos $(>60 \text { anos })^{10}$, resultando em: $1.663 \mathrm{kcal} / \mathrm{dia}$; 210,78 g/dia de carboidratos; 64,95 g/dia de proteínas e 61,66 g/dia de lipídios.

\section{Análise estatística}

Os dados foram avaliados por meio da análise de variância (ANOVA), utilizando-se o teste de Tukey e t de student para comparação de médias. Na análise sensorial foram empregados também, o Teste de Friedman e a Tabela de Christensen que indicaram a diferença mínima significativa (DMS) entre as amostras testadas e o número de julgamentos obtidos no teste de ordenação ${ }^{6}$. Todos os testes foram analisados em nível de $5 \%$ de significância, com auxílio do software Statgraphics plus, versão 5.1.

\section{Questões éticas}

Este trabalho foi aprovado pelo Comitê de Ética em Pesquisa da UNICENTRO, parecer número $n^{\circ}$ 262/2011. Como critérios de exclusão foram considerados os seguintes fatores: indivíduos que não possuíam Diabetes Mellitus, aqueles que não aceitaram participar da pesquisa e que não assinaram o Termo de Consentimento Livre e Esclarecido (TCLE). 
Rodrigues MGG, Santos EF, Sanches FLFZ, Novello D, Manhani MR, Neumann M. Desenvolvimento de cookies adicionados de farinha de yacon (Smallanthus sonchifolius): caracterização química e aceitabilidade sensorial entre portadores de Diabetes Mellitus Rev Inst Adolfo Lutz. São Paulo, 2014; 73(2):219-25.

Tabela 1. Médias dos testes sensorial afetivo, ordenação, intenção de compra e índice de aceitabilidade (IA), realizados para as formulações de cookies padrão (0 \%) e adicionados de 5,0; 7,5; 10,0 e 12,5\% de farinha de yacon (FY)

\begin{tabular}{|c|c|c|c|c|c|}
\hline \multirow{2}{*}{ Atributos } & \multicolumn{5}{|c|}{ Média \pm Erro padrão da média } \\
\hline & F1 & F2 & F3 & F4 & F5 \\
\hline Aparência & $7,30 \pm 0,12^{\mathrm{a}}$ & $6,90 \pm 0,18^{\mathrm{ab}}$ & $6,74 \pm 0,19^{\mathrm{abc}}$ & $6,01 \pm 0,08^{\mathrm{bc}}$ & $5,81 \pm 0,11^{\mathrm{c}}$ \\
\hline IA (\%) & 81,11 & 76,67 & 74,89 & 66,78 & 64,56 \\
\hline Aroma & $7,34 \pm 0,15^{\mathrm{a}}$ & $7,29 \pm 0,16^{\mathrm{a}}$ & $6,52 \pm 0,13^{\mathrm{ab}}$ & $6,08 \pm 0,14^{\mathrm{b}}$ & $6,06 \pm 0,14^{\mathrm{b}}$ \\
\hline Sabor & $7,94 \pm 0,12^{\mathrm{a}}$ & $7,61 \pm 0,14^{\mathrm{a}}$ & $6,92 \pm 0,17^{\mathrm{a}}$ & $5,76 \pm 0,18^{\mathrm{b}}$ & $5,87 \pm 0,17^{\mathrm{b}}$ \\
\hline IA (\%) & 88,22 & 84,56 & 76,89 & 64,00 & 65,22 \\
\hline Textura & $7,65 \pm 0,20^{\mathrm{a}}$ & $6,87 \pm 0,10^{\mathrm{ab}}$ & $6,54 \pm 0,15^{\mathrm{b}}$ & $5,24 \pm 0,14^{c}$ & $5,40 \pm 0,11^{c}$ \\
\hline IA (\%) & 85,44 & 82,78 & 70,44 & 71,11 & 70,67 \\
\hline Aceitação Global & $7,80 \pm 0,15^{\mathrm{a}}$ & $7,36 \pm 0,19^{\mathrm{a}}$ & $6,72 \pm 0,21^{\mathrm{ab}}$ & $5,63 \pm 0,18^{\mathrm{bc}}$ & $5,54 \pm 0,19^{c}$ \\
\hline IA (\%) & 86,67 & 81,78 & 74,67 & 62,56 & 61,56 \\
\hline Intenção de Compra & $4,09 \pm 0,12^{\mathrm{a}}$ & $3,69 \pm 0,10^{\mathrm{a}}$ & $3,87 \pm 0,12^{\mathrm{a}}$ & $2,47 \pm 0,15^{b}$ & $2,50 \pm 0,16^{\mathrm{b}}$ \\
\hline Somatório das notas ${ }^{\star}$ & $235^{\mathrm{a}}$ & $211^{\mathrm{a}}$ & $171^{\mathrm{b}}$ & $117^{\mathrm{c}}$ & $91^{\mathrm{c}}$ \\
\hline
\end{tabular}

\section{RESULTADOS}

\section{Análise Sensorial}

A Tabela 1 apresenta as médias verificadas para o teste sensorial afetivo, de intenção de compra e índice de aceitabilidade dos biscoitos tipo cookies padrão e enriquecidos com FY.

A adição de FY aos cookies nas porcentagens de 10,0 e $12,5 \%$ promoveu uma menor aceitação para os atributos aparência, sabor e aroma $(p<0,05)$. Avaliandose $\mathrm{o}$ atributo textura, foi possível observar que as amostras F1 e F2 apresentaram as maiores notas seguidas por F3 $(p<0,05)$, enquanto $\mathrm{F} 4 \mathrm{e} 55$ foram aquelas menos referidas para este atributo $(p<0,05)$. Ressalta-se que produtos com maiores porcentagens de FY foram referidos pelos provadores com uma consistência mais "rígida".

As formulações F1 e F2 apresentaram as maiores notas para o atributo cor, quando comparadas a F4 e F5, sendo que F3 não mostrou diferença significativa das demais $(p>0,05)$. Durante a elaboração dos cookies, verificou-se que as formulações com maiores teores de FY apresentaram coloração mais escura.

Os julgadores relataram maior aceitação global para as formulações F1 e F2, que decresceu com a adição de maiores níveis de FY $(p<0,05)$. As amostras F1, F2 e F3 apresentaram as melhores referências para intenção de compra $(p>0,05)$, que também reduziu a preferência conforme a porcentagem de adição de FY aumentou.

As formulações F1, F2 e F3 apresentaram índice de aceitabilidade acima de $70 \%$, que os classifica como produtos de boa aceitação sensorial ${ }^{11}$. No teste de ordenação, as formulações F1 e F2 foram relatadas pelos provadores como sendo as mais doces, seguidas por F3 $(p<0,05)$. Os produtos F4 e F5 foram descritos com o menor teor de doçura, sendo estas as formulações que continham as mais baixas concentrações de açúcar. 
Rodrigues MGG, Santos EF, Sanches FLFZ, Novello D, Manhani MR, Neumann M. Desenvolvimento de cookies adicionados de farinha de yacon (Smallanthus sonchifolius): caracterização química e aceitabilidade sensorial entre portadores de Diabetes Mellitus Rev Inst Adolfo Lutz. São Paulo, 2014; 73(2):219-25.

Sendo os atributos aroma e sabor considerados como aqueles que mais influenciam as propriedades sensoriais de produtos alimentícios adicionados de ingredientes diferenciados, a amostra F3 (7,5 \%) foi selecionada para fins de comparação, juntamente com a padrão (F1), por ser aquela com o maior teor de FY e com aceitação semelhante a padrão.

\section{Análises químicas}

A Tabela 2 apresenta a composição química e os valores diários recomendados (VD) dos cookies padrão e dos cookies adicionados de 7,5 \% de FY, em comparação a um produto referência.

Foram observados maiores teores $(p<0,05)$ de umidade, cinzas, proteínas e fibra bruta para os biscoitos contendo FY (F3), sendo que, em geral, os valores foram superiores aos do produto referência ${ }^{12}$. Entretanto, a composição de carboidratos e calorias dos cookies padrão foi maior que em F3 $(p<0,05)$. Não houve diferença significativa nos conteúdos de lipídios entre as formulações F1 e F3, sendo resultados também maiores que o biscoito referência ${ }^{12}$.

\section{DISCUSSÃO}

\section{Análise sensorial}

Resultados semelhantes aos apresentados na Tabela
1 foram observados por Rolim et $\mathrm{al}^{3}$ que, ao avaliarem formulações de pães acrescidos de FY (0, 6,0 e 11,0 \%), verificaram que a maior porcentagem de adição do ingrediente também levou à menor aceitação do produto.

A consistência mais "rígida" referida aos produtos com maiores porcentagens de FY pode ser explicada pelo conteúdo de inulina e FOS da yacon e, por apresentar grupos de $\mathrm{OH}^{-}$disponíveis, capazes de interagir com moléculas de água por pontes de hidrogênio e, desta forma, dificultar sua evaporação durante o processo de cocção, tornando o produto menos crocante. Resultados similares foram verificados por Moscatto et $\mathrm{al}^{13}$, ao elaborarem bolos de chocolate adicionados de 20 e $40 \%$ de FY em substituição à farinha de trigo.

A diferença de textura entre os produtos também pode ser explicada pelo fato das formulações F1 e F2 conterem maiores teores de sacarose, o qual é sabido estar diretamente relacionado à melhoria das características da massa dos biscoitos para conferir maior maciez ao produto $^{14}$. Entretanto, o açúcar é um alimento de alto índice glicêmico, que pode elevar rapidamente a glicose sanguínea e prejudicar os pacientes com $\mathrm{DM}^{4}$. Desta forma, quanto menor for o consumo deste ingrediente por indivíduos diabéticos, maiores serão os benefícios à saúde.

A coloração mais escura dos cookies adicionados de maiores teores de FY se deve, principalmente, a cor

Tabela 2. Composição química e valores diários recomendados - VD* (porção média de 30 gramas) de cookies padrão (F1) e adicionados de 7,5 $\%$ de farinha de yacon (F3), comparadas com um produto referência**

\begin{tabular}{|c|c|c|c|c|c|}
\hline \multirow{2}{*}{ Avaliação } & \multicolumn{2}{|c|}{ F1 } & \multicolumn{2}{|c|}{ F3 } & \multirow{2}{*}{ Referência $^{* *}$} \\
\hline & Média $\pm \mathrm{DP}$ & $\operatorname{VD}(\%)^{*}$ & Média $\pm D P$ & VD $(\%)^{*}$ & \\
\hline Umidade (\%) & $9,54 \pm 0,02^{\mathrm{b}}$ & ND & $15,00 \pm 0,08^{\mathrm{a}}$ & ND & 8,00 \\
\hline Cinzas $\left(\mathrm{g} .100 \mathrm{~g}^{-1}\right)$ & $0,88 \pm 0,01^{\mathrm{b}}$ & ND & $1,14 \pm 0,00^{\mathrm{a}}$ & ND & 0,95 \\
\hline Proteínas (g.100g $\left.{ }^{-1}\right)$ & $8,20 \pm 0,21^{\mathrm{b}}$ & 3,79 & $9,30 \pm 0,03^{\mathrm{a}}$ & 4,30 & 4,20 \\
\hline Lipídios $\left(\mathrm{g} \cdot 100 \mathrm{~g}^{-1}\right)$ & $6,54 \pm 0,07^{\mathrm{a}}$ & 3,18 & $6,11 \pm 0,09^{a}$ & 2,97 & 2,70 \\
\hline Carboidratos $\left(\mathrm{g} .100 \mathrm{~g}^{-1}\right)$ & $74,85 \pm 0,26^{\mathrm{a}}$ & 10,65 & $68,45 \pm 0,18^{\mathrm{b}}$ & 9,74 & 84,00 \\
\hline Calorias (kcal.100 $\left.\mathrm{g}^{-1}\right)$ & $394,08 \pm 0,06^{\mathrm{a}}$ & 7,11 & $368,47 \pm 010^{\mathrm{b}}$ & 6,65 & 378,00 \\
\hline Fibra bruta $\left(\mathrm{g} .100 \mathrm{~g}^{-1}\right)$ & $7,90 \pm 0,03^{\mathrm{b}}$ & ND & $10,57 \pm 0,10^{\mathrm{a}}$ & ND & ND \\
\hline
\end{tabular}

Letras diferentes na linha indicam diferença significativa pelo teste de t de student $(p<0,05) ;{ }^{\star} \mathrm{VD}$ : nutrientes avaliados pela média da DRI ${ }^{10}$, com base numa dieta de $1.663 \mathrm{kcal} / \mathrm{dia}$; ${ }^{\star *}$ Valores comparados com "biscoito cookies tradicional”"2; Valores calculados em base úmida; DP: desvio padrão da média; ND: não disponível. 
Rodrigues MGG, Santos EF, Sanches FLFZ, Novello D, Manhani MR, Neumann M. Desenvolvimento de cookies adicionados de farinha de yacon (Smallanthus sonchifolius): caracterização química e aceitabilidade sensorial entre portadores de Diabetes Mellitus Rev Inst Adolfo Lutz. São Paulo, 2014; 73(2):219-25.

marrom característica do processo de escurecimento enzimático da yacon. Também, uma das funções do açúcar é melhorar a cor e aparência dos produtos durante a cocção, sendo considerado um corante natural ${ }^{14}$. Nesse sentido, maiores porcentagens de adição de FY podem ter reduzido as notas para este atributo.

Avaliando-se o teste de ordenação para o sabor doce, ressalta-se que os biscoitos poderão ter seu sabor aprimorado tecnologicamente, pelo uso de edulcorantes naturais e/ou artificiais, melhorando assim este atributo sensorial sem prejudicar a saúde dos pacientes portadores de DM.

\section{Análises químicas}

Quanto ao teor de umidade dos produtos, constatou-se que apenas a formulação padrão estava de acordo com a recomendação da RDC 12/1978, da Agência Nacional de Vigilância Sanitária (ANVISA), a qual define o máximo de $14 \%$ de umidade em biscoitos e bolachas ${ }^{15}$.

O maior conteúdo de cinzas obtido em F3 pode estar relacionado ao fato da yacon possuir considerável teor de minerais $(3,63 \%)^{16}$, enquanto que o mais elevado de teor protéico verificado para F3 está diretamente relacionado à presença de proteínas na farinha de yacon $(2,79 \%)^{16}$. Ressalta-se, ainda, que as maiores quantidades desse macronutriente, verificadas em F1 e F3 quando comparado ao produto referência ${ }^{12}$, provavelmente, se deveram aos diferentes ingredientes utilizados nas formulações.

A redução nos teores de carboidratos e calorias verificada para os produtos contendo FY ocorreu principalmente, devido ao fato da formulação F3 ter sido elaborada com teor reduzido de açúcar, o qual contém maior quantidade energética proveniente dos carboidratos. Além disso, a FY possui $42,52 \%$ de carboidratos complexos na sua composição ${ }^{16}$, quando comparada à sacarose (5 a $15 \%$ ), considerada como carboidrato simples e que deve ser reduzida na dieta de indivíduos com glicemia alterada ${ }^{4}$. Dessa forma, os cookies com adição de FY podem ser considerados alimentos alternativos para pacientes portadores de DM, considerando o baixo índice glicêmico apresentado pela yacon. Entretanto, ressalta-se que é necessária moderação no consumo, uma vez que a sacarose, mesmo que em menor quantidade, ainda está presente na formulação. Destaca-se que a redução de aproximadamente $6 \%$ de calorias pode colaborar no tratamento de pacientes de
$\mathrm{DM}$, que necessitam de um menor consumo calórico em sua dieta ${ }^{4}$.

Enfatiza-se como o principal resultado desse trabalho o aumento significativo de fibra bruta $(33,80$ $\%)$ em relação a $\mathrm{F} 1$. Isso se deve, principalmente, ao alto teor de fibra solúvel e insolúvel $\left(2,24\right.$ e 15,91 g. $100 \mathrm{~g}^{-1}$, respectivamente) presente na $\mathrm{FY}^{16}$. Estes resultados tornam o produto uma excelente opção para portadores de $\mathrm{DM}$, pois as fibras auxiliam no retardo da absorção de carboidratos, levando à diminuição da glicemia sanguínea ${ }^{4}$.

De acordo com a Legislação Brasileira ${ }^{17}$, um produto é considerado como fonte de fibra alimentar quando apresentar no mínimo $3 \%$ e com alto teor no mínimo $6 \%$ em fibras. Assim, sabendo-se que o método de determinação de fibra bruta utilizado no presente trabalho subestima o valor de fibra alimentar nos produtos, pode-se considerar ambas as formulações com alto teor em fibras.

\section{CONCLUSÃO}

O desenvolvimento dos produtos permitiu, neste estudo, comprovar que um nível de adição de até 7,5\% de farinha de yacon em biscoitos tipo cookies foi aceito pelos provadores, com aceitação sensorial semelhante ao produto padrão.

A adição de $7,5 \%$ de farinha de yacon em biscoitos proporcionou, em geral, aumento no teor de nutrientes, com exceção dos carboidratos e calorias. Foi possível também, elevar o aporte de fibras, melhorando o perfil nutricional do produto.

Assim sendo, a farinha de yacon pode ser considerada como potencial ingrediente com propriedades funcionais a ser adicionados em biscoitos e similares, a serem oferecidos aos consumidores com boas expectativas de aceitação no mercado.

\section{REFERÊNCIAS}

1. Mclellan KCP, Barbalho SM, Cattalini M, Lerario AC. Diabetes mellitus do tipo 2, síndrome metabólica e modificação no estilo de vida. Rev Nut.2007;20(5):515-24.

2. Santana I, Cardoso MH. Raiz tuberosa de yacon (Smallanthus sonchifolius): potencialidade de cultivo, aspectos tecnológicos e nutricionais. Ciênc Rural.2008;38(3):898-905.

3. Rolim PM, Salgado SM, Padilha VM, Livera AVS, Guerra NB, Andrade SAC. Análise de componentes principais de pães de forma formulados com farinha de yacon (Smallanthus sonchifolius (Poepp.) H. Rob.). Rev Ceres.2010;57(1):12-7. 
Rodrigues MGG, Santos EF, Sanches FLFZ, Novello D, Manhani MR, Neumann M. Desenvolvimento de cookies adicionados de farinha de yacon (Smallanthus sonchifolius): caracterização química e aceitabilidade sensorial entre portadores de Diabetes Mellitus Rev Inst Adolfo Lutz. São Paulo, 2014; 73(2):219-25.

4. Martins MLR, Delmaschio KL, Cordeiro AA. Efeitos da utilização de Smallanthus sonchifolius (yacon) no tratamento de indivíduos com Diabetes Mellitus. Rev Ceres.2011;6(1):35-43.

5. Instituto Adolfo Lutz. Normas analíticas do Instituto Adolfo Lutz: Métodos químicos e físicos para análises de alimentos. $4^{\mathrm{a}}$ ed. Brasília (DF): IAL; 2008.

6. Dutcosky SD. Análise Sensorial de Alimentos. $3^{\text {a }}$ ed. Curitiba (PR): Champagnat; 2011.

7. Bligh EG, Dyer WJ. A rapid method of total lipid extraction and purification. Can J Biochem Physiol.1959;37(8):911-7.

8. Merrill AL, Watt BK. Energy values of foods: basis and derivation. Agricultural Handbook, n.74, Washington (DC): USDA; 1973.

9. Brasil. Ministério da Saúde (MS). Agência Nacional de Vigilância Sanitária (ANVISA). Rotulagem nutricional obrigatória: manual de orientação às indústrias de Alimentos - 2º Versão. Brasília (DF): ANVISA/Universidade de Brasília; 2005.

10. Dietary Reference Intakes (DRI) for energy, carbohydrate, fiber, fat, fatty acids, cholesterol, protein and amino acids. Washington (DC): National Academy Press; 2000.

11. Teixeira E, Meinert E, Barbetta PA. Análise Sensorial dos Alimentos. Florianópolis: UFSC; 1987.

12. United States Department of Agriculture (USDA). Nutrient Database for Standard Reference. 2001. [acesso 2012 jul 28]. Disponível em: [http://www.unifesp.br/dis/ servicos/nutri/nutri. hp?d=101].
13. Moscatto JA, Borsato D, Bona E, Oliveira AS, Hauly COM. The optimization of the formulation for a chocolate cake containing inulin and yacon meal. Int J Food Sci Technol.2005;41(2):181-8.

14. Ornellas LH. Técnica Dietética - seleção e preparo de alimentos. $8^{a}$ ed. São Paulo (SP): Atheneu; 2007.

15. Brasil. Resolução n. 12, de julho de 1978. Aprova Normas Técnicas Especiais, do Estado de São Paulo, revistas pela CNNPA, relativas a alimentos (e bebidas), para efeito em todo território brasileiro. Diário Oficial [da] República Federativa do Brasil. Brasília, DF, 23 jul. 1978.

16. Vasconcelos CM, Silva CO, Teixeira LJQ, Chaves JBP, Martino HSD. Determinação da fração da fibra alimentar solúvel em raiz e farinha de yacon (Smallanthus sonchifolius) pelo método enzimático-gravimétrico e cromatografia líquida de alta eficiência. Rev Inst Adolfo Lutz.2010;69(2):188-93.

17. Brasil. Agência Nacional de Vigilância Sanitária. ANVISA. Resolução da Diretoria Colegiada - RDC No 54, de 12 de novembro de 2012. Aprova: "Regulamento Técnico sobre Informação Nutricional”. [acesso 2014 jun 11]. Disponível em: [http://portal. anvisa.gov.br/wps/wcm/connect/630a988 04d7065b981f1e1c116238c3b/Resolucao+RDC+n.+54_2012. pdf?MOD=AJPERES]. 